\title{
Fabrication of Solidified Ionic Liquid with 3D Microstructures and Its Application to Vibration Energy Harvester
}

\author{
Taiki Iida, ${ }^{1}$ Takuya Tsukamoto, ${ }^{1}$ Kazumoto Miwa, ${ }^{2}$ \\ Shimpei Ono, ${ }^{2,3}$ and Takaaki Suzuki ${ }^{1,3 *}$ \\ ${ }^{1}$ Gunma University, 1-5-1 Tenjin cho, Kiryu-shi, Gunma 376-8515, Japan \\ ${ }^{2}$ Central Research Institute of Electric Power Industry, 2-6-1 Nagasaka, Yokosuka, Kanagawa 240-0196, Japan \\ ${ }^{3}$ JST PRESTO, 4-1-8 Honcho, Kawaguchi, Saitama 332-0012, Japan \\ (Received January 23, 2019; accepted April 9, 2019)
}

Keywords: vibration energy harvester, photolithography, 3D microfabrication, ionic liquid, solidified ionic gel, micro-electromechanical systems

In this study, we propose a solidified ionic liquid with a 3D microstructure to increase its surface area for the performance enhancement of a vibration energy harvester (VEH). By soft lithography in MEMS technologies, the use of a mold is proposed to perform the solidification, polarization, and microstructure transfer of the solidified ionic liquid simultaneously. We fabricated six samples with different surface shapes and sizes to compare the power generation performance characteristics of VEHs using a solidified ionic liquid. According to a vibration test, the performance of the VEH with nanometer roughness was improved at a $10 \mathrm{~Hz}$ frequency. Also, the output power of the VEH with a micro-folded hollow conical structure was improved at a $50 \mathrm{~Hz}$ frequency.

\section{Introduction}

Along with the progress of the Internet of Things (IoT) society, power supply to a massive number of sensor nodes, the battery replacement, and the maintenance cost have been major issues. $^{(1,2)}$ Thus, the power supply is desired to be self-sustainable. As an alternative solution to the issues, energy harvesting, which obtains electric power from external energy sources, has attracted considerable attention. ${ }^{(3-7)}$ Among energy sources, vibration is potentially a more extractable energy than other sources such as sunlight, radio waves, and heat. ${ }^{(3)}$ In addition, as vibration sources exist everywhere, there are various applications of vibration energy harvesting, e.g., wearable devices, maintenance for buildings and gas pipelines, and automotive sensors. ${ }^{(9,10)}$

In recent years, an ionic liquid has attracted attention as a power generation material because it has excellent electric characteristics. ${ }^{(11)}$ In addition, a vibration energy harvester (VEH) using solidified ionic liquids with electric double layers has been reported recently. ${ }^{(12)}$ Multiple factors, i.e., piezoelectricity, triboelectricity, and electrostatic induction, are considered to affect

*Corresponding author: e-mail: suzuki.taka@gunma-u.ac.jp

https://doi.org/10.18494/SAM.2019.2309 
the power generation mechanism of a solidified ionic liquid. For all generation mechanisms, the power generation performance of the VEH increases with the surface area of a solidified ionic liquid.

Therefore, in this study, we increase the surface area of a solidified ionic liquid by forming a 3D microstructure to improve the output power without increasing the footprint of the VEH. We propose a novel forming method to simultaneously perform the solidification, polarization, and microstructure transfer of the solidified ionic liquid using a mold with 3D micropatterns fabricated by 3D lithography. ${ }^{(13-15)}$ The power generation performance characteristics of the fabricated VEHs were evaluated by vibration tests.

\section{Principle of VEH}

The power generation principle of the proposed VEH is shown in Fig. 1. Anions or cations are fixed on the surface of the solidified ionic liquid by polarization. When the surface of the solidified ionic liquid is in contact with the upper electrode, an approximately $1 \mathrm{~nm}$ electric double-layer capacitor with a large electric capacitance is formed at the contact interface. ${ }^{(11)}$ Then, the capacitance largely changes in accordance with the change in contact surface area between the solidified ionic liquid and the electrode. At that time, the electric charge moves and the current flows through a connected external load resistance. In this study, a 3D microstructure is considered to possibly fix more electric charges and improve the power generation performance by increasing the surface area of the solidified ionic liquid.

\section{Fabrication of VEH}

The proposed VEH is fabricated by solidifying an ionic liquid using a mold with a flat surface, a nanosized unevenness, or a 3D microstructure. In this section, the solidification of the ionic liquid and the fabrication of a mold with a 3D microstructure are described in detail.
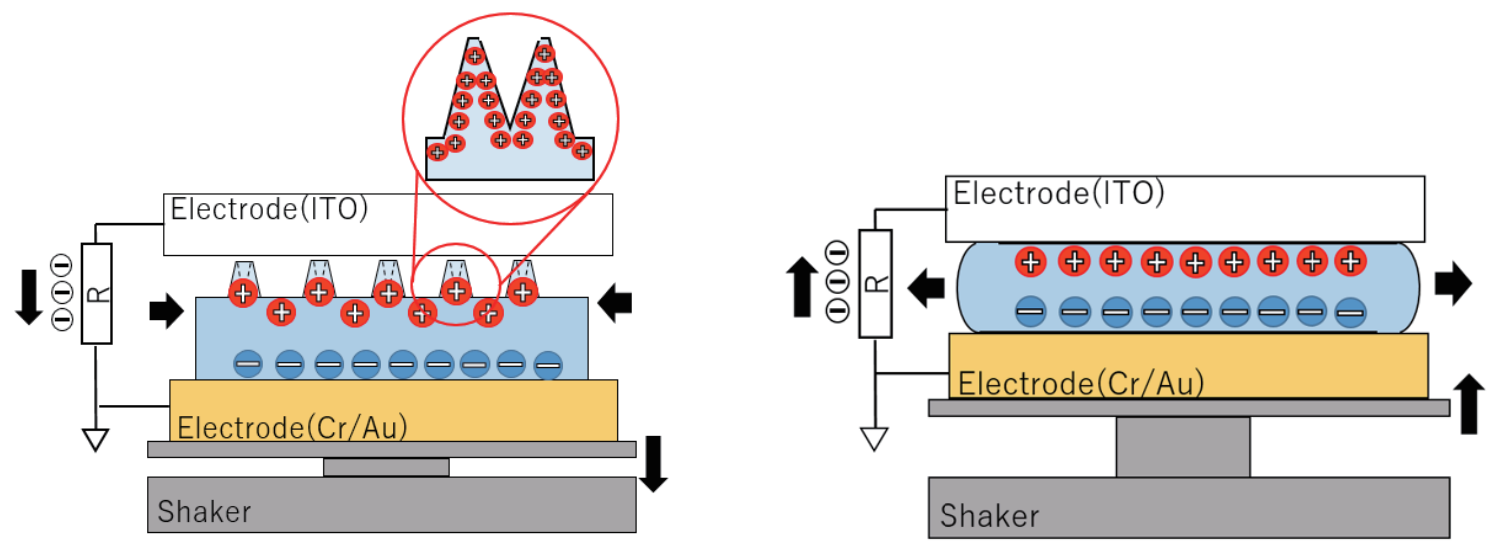

Fig. 1. (Color online) Principle of power generation. 


\subsection{Solidification of ionic liquid with 3D microstructure}

An ionic liquid with a high electrophoretic property is polarized by applying voltage. A macromonomer with a crosslinking function and an ionic liquid are mixed with a photopolymerization initiator and solidified by irradiating ultraviolet light. Here, the chain polymer of the monomer part is crosslinked. ${ }^{(12)}$ The shape of the solidified ionic liquid is formed by pouring the ionic liquid into a mold with an arbitrary shape. In this study, 3D microstructures are transferred from a mold by soft lithography in MEMS fabrication technologies. Since the proposed mold has microstructures and electrodes, all of the solidification, polarization, and transfer of microstructures can be simultaneously performed by irradiating UV light and applying voltage.

Figure 2 shows a schematic diagram of the solidification process. The solidification by using the mold with 3D microstructures was processed in a glove box filled with argon gas because the ionic liquid absorbs moisture from the atmosphere. A mixture of an acrylic polymer, an ionic liquid with an unsaturated bond, and a photopolymerization initiator (1-hydroxycyclohexyl-phenyl ketone) with a weight ratio of 100:10:1 was dropped onto the mold. The whole size and shape of the solidified ionic liquid were determined using a polytetrafluoroethylene (PTFE) spacer with a hole of $500 \mu \mathrm{m}$ thickness. Then, the ionic liquid was vacuum-cast-molded in the pattern area. Next, the ionic liquid was sandwiched between an indium-tin oxide (ITO) electrode on a glass substrate and the mold with a Au electrode. DC $4 \mathrm{~V}$ was applied for $5 \mathrm{~min}$ to polarize the ionic liquid. Then, to complete the solidification and polarization of the ionic liquid, UV light with an intensity of $11.52 \mathrm{~J} / \mathrm{cm}^{2}$ was irradiated from the top ITO glass side while applying the DC voltage for the polarization.

The six different solidified ionic liquids shown in Table 1 were prepared to compare their power generation performance characteristics due to the change in surface structure. The six samples are classified as two flat disks (samples \#1 and \#2) with different diameters and four disks with a surface fine structure of nanosized unevenness (sample \#3), a microfolded hollow conical structure (sample \#4), a microcylindrical structure (sample \#5), and a microconical

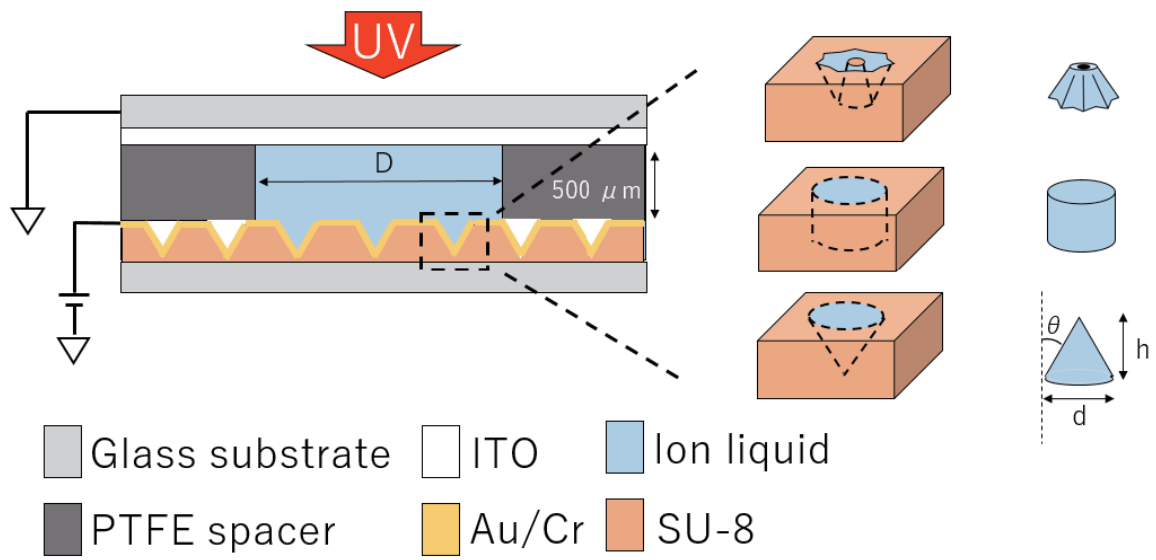

Fig. 2. (Color online) Schematic of fabrication process for solidified ionic liquid. 
Table 1

Dimensions of fabricated solidified ionic liquid.

\begin{tabular}{lccccc}
\hline Sample\# & $D(\mathrm{~mm})$ & Structure & $d(\mu \mathrm{m})$ & $h(\mu \mathrm{m})$ & $\theta\left(^{\circ}\right)$ \\
\hline 1 & 3 & $\begin{array}{c}\text { Flat } \\
\text { (No structure) }\end{array}$ & - & - & - \\
\hline 2 & 5 & $\begin{array}{c}\text { Flat } \\
\text { (No structure) }\end{array}$ & - & - & - \\
\hline 3 & 3 & Nanosized unevenness & - & 15 & - \\
\hline 4 & 3 & $\begin{array}{c}\text { Microfolded hollow } \\
\text { conical structure }\end{array}$ & 48 & 41 & 30 \\
\hline 5 & 3 & Microcylindrical structure & 48 & 41 & 30 \\
\hline 6 & 3 & Microconical structure & 48 & - & 0 \\
\hline
\end{tabular}

structure (sample \#6). Among those structures, the microfolded hollow conical structure has a particularly larger surface area than the others. Therefore, it fixes more electrical charges on the surface. Moreover, it is expected to increase the contact area with an electrode because it is more flexible.

\subsection{Fabrication of mold with 3D microstructures and electrode}

A transfer mold with both 3D microstructures and an electrode was made of a thick film photoresist. The sample with nanosized unevenness was prepared by oxygen ashing ${ }^{(16)}$ and the samples with a microsized 3D structure were formed by 3D lithography. ${ }^{(13-15)}$

In the 3D lithography method, both a photoresist-coated substrate and a photomask are fixed to an adjustable table that controls the table angle and rotation speed. The substrate is exposed from an inclination direction while rotating at a constant speed. The UV light is irradiated vertically to the horizontal surface of the inclined/rotated table, i.e., irradiated obliquely to the photoresist as shown in Fig. 3. Thus, the UV light refracts between the two media according to Snell's law. By rotating the inclined table during the exposure, the direction of the incident UV light to the photoresist is continuously changed. Therefore, 3D microstructures with complex shapes in the out-of-plane direction, which cannot be formed by conventional vertical exposure, can be produced. Here, the angle $\theta r$ of the inclined structure shown in Fig. 3(b) is determined as

$$
\theta_{r}=\sin ^{-1}\left(\frac{n}{n_{r}} \sin \theta_{0}\right),
$$

where the refractive indexes of air, photomask glass, and photoresist are $n, n_{m}$, and $n_{r}$, respectively. The inclination angle of the rotating inclined table, i.e., the incident angle of UV to the photomask, is $\theta_{0}$, and the refraction angles in the photomask and photoresist are $\theta_{m}$ and $\theta_{r}$, respectively. By substituting the refractive indices of SU-8 1.6 and air 1.0 into Eq. (1), the structure with an inclination angle of $30^{\circ}$ used in this study was prepared by setting the inclination angle to $53.1^{\circ}$. 


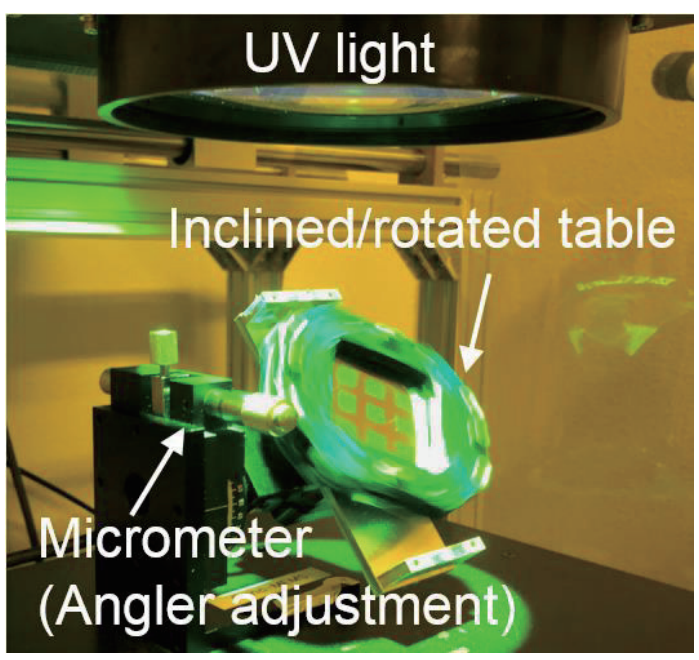

(a)

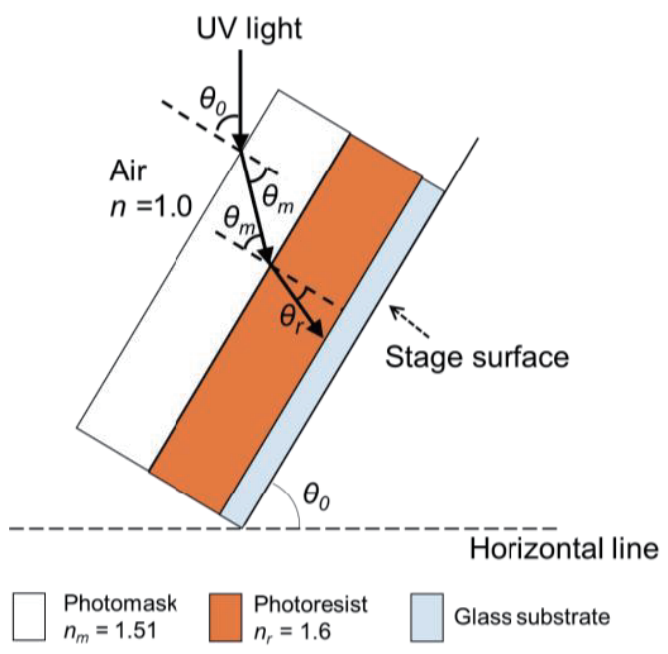

(b)

Fig. 3. (Color online) Angular control of 3D lithography: (a) photograph of exposure apparatus and (b) refraction of UV light between the photomask and the photoresist.

As shown in Fig. 4, a transfer mold with a 3D microstructure and an electrode was fabricated by 3D lithography. First, an oligomeric adhesion promotor (OAP) (Tokyo Ohka Kogyo Co., Ltd.) was spin-coated on a glass substrate and its surface was treated with hexamethyldisilazane (HDMS) to improve the adhesion between the glass substrate and the photoresist. Then, the thick-film negative resist SU-8 3050 (Micro Chem Corp.) was formed by spin coating and baked on a hot plate at $95{ }^{\circ} \mathrm{C}$ for $2 \mathrm{~h}$. Figure 5 shows a photomask pattern for the lithography. The micropatterns with a size of $48 \mathrm{~mm}$ were arrayed with a gap of $52 \mathrm{~mm}$. In the exposure process, the thick negative photoresist SU-8 was exposed by vertical or 3D lithography while contacting the photomask under each condition as shown in Table 2. After the exposure, SU-8 was subjected to post-exposure baking (PEB) at $95{ }^{\circ} \mathrm{C}$ for $8 \mathrm{~min}$. Since the entire surfaces of samples \#1 and \#2 with nanosized unevenness were exposed, wet development became unnecessary and oxygen ashing (oxygen gas flow rate: $110 \mathrm{sccm}$, pressure: $40 \mathrm{~Pa}, \mathrm{RF}$ output: $200 \mathrm{~W}$, and ashing time: $10 \mathrm{~min}$ ) was performed using a reactive ion etching machine (ES 401, Nippon Scientific Co., Ltd.) to form nanosized unevenness on the SU-8 surface. Samples \#3-\#6 with 3D microstructures were wet-developed in PGMEA (1-methoxy-2-propyl acetate, SU-8 developer, Micro Chem Corp.) for $18 \mathrm{~min}$ to form the structure.

Finally, a Cr film with a thickness of $30 \mathrm{~nm}$ and a Au film with a thickness of $170 \mathrm{~nm}$ were deposited on the micro- or nanostructured surfaces of the samples by sputtering. By using the Au film as an electrode on the samples, an ionic liquid was solidified as shown in Fig. 2. The SEM images of the transferred 3D microstructures made of solidified ionic liquid are shown in Fig. 6. It can be seen that the 3D microstructures of the mold were transferred to the surface of the solidified ionic liquid. 


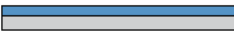

(1) Primer spin coating

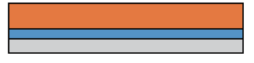

(2) SU-8 spin coating

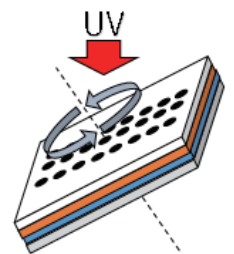

(3) Inclined/rotated exposure

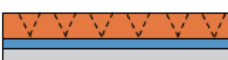

(4) SU-8 developing

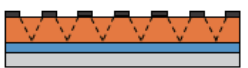

(5) Cr sputtering

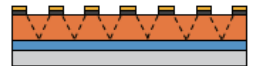

(6) Au sputtering

Glass substrate Primer SU-8 $\square$ Photomask cr $\square$ Au

Fig. 4. (Color online) Fabrication process for a mold with a 3D microstructure.
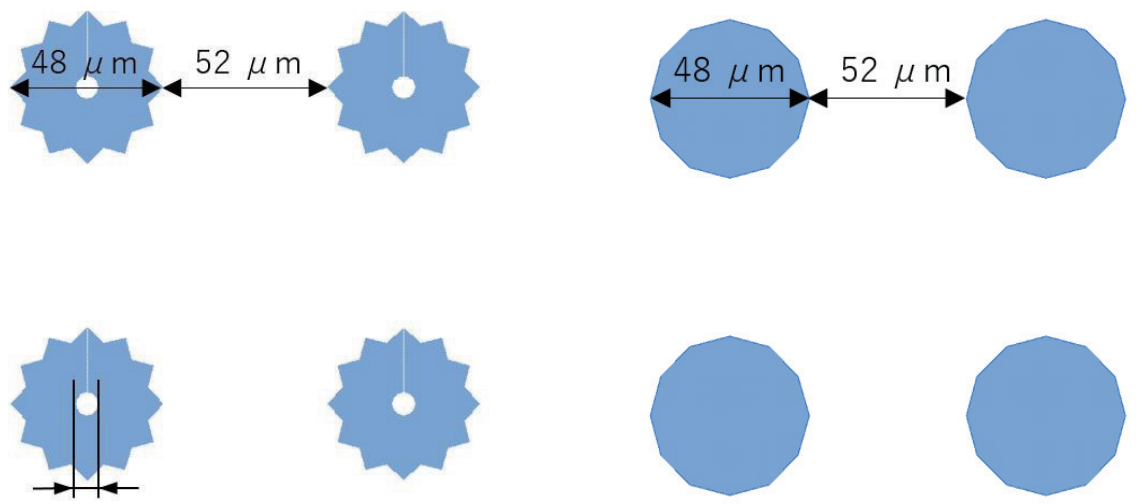

(a)

(b)

Fig. 5. (Color online) Photomask designs: (a) polygon and (b) circular patterns.

Table 2

Exposure conditions.

\begin{tabular}{lccc}
\hline Structure & Exposure method & Photomask & $\begin{array}{c}\text { Exposure dose } \\
\left(\mathrm{mJ} / \mathrm{cm}^{2}\right)\end{array}$ \\
\hline Nanosized unevenness & Conventional exposure & None & 150 \\
\hline $\begin{array}{l}\text { Microfolded hollow } \\
\text { conical structure }\end{array}$ & 3D lithography & Fig. 5(a) & 400 \\
\hline Microcylindrical structure & Conventional exposure & Fig. 5(b) & 150 \\
\hline Microconical structure & 3D lithography & Fig. 5(b) & 400 \\
\hline
\end{tabular}




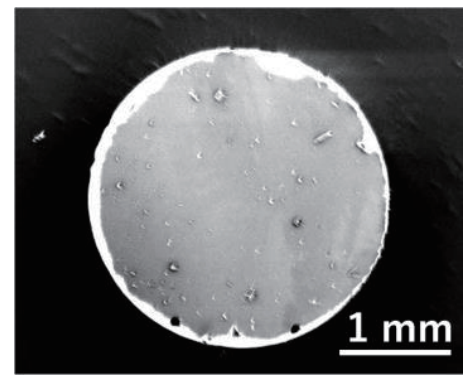

(a)

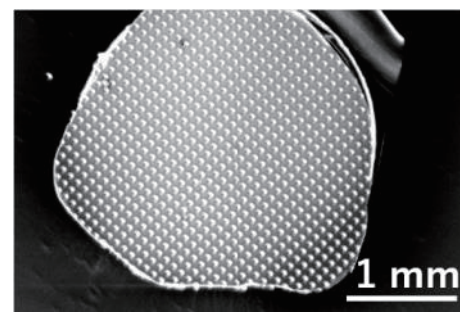

(c)

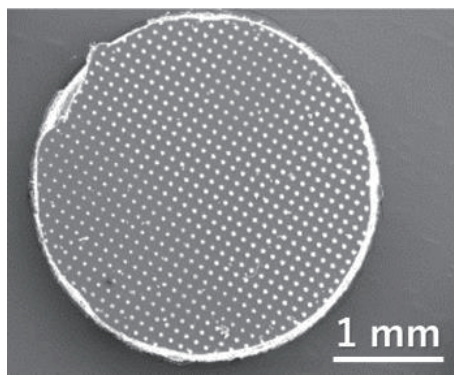

(e)

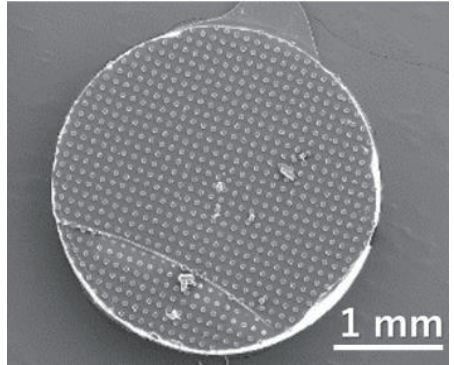

(g)

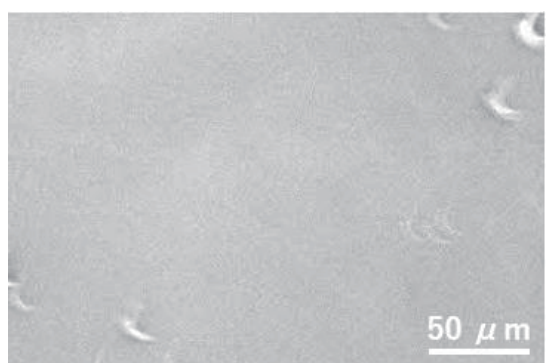

(b)

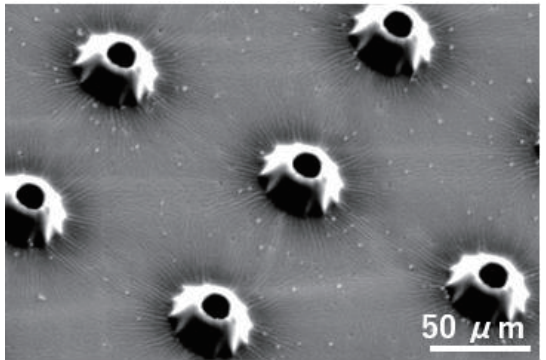

(d)

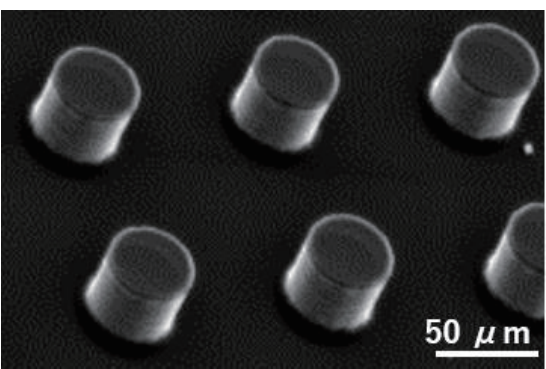

(f)

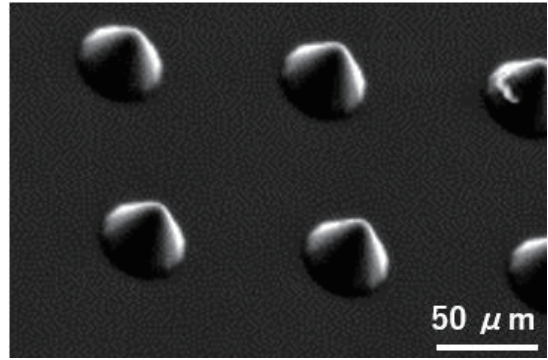

(h)

Fig. 6. SEM images of the fabricated solidified ionic liquid: (a) whole image of sample \#1, (b) enlarged image of sample \#1, (c) whole image of sample \#4, (d) enlarged image of sample \#4, (e) whole image of sample \#5, (f) enlarged image of sample \#5, (g) whole image of sample \#6, and (h) enlarged image of sample \#6. 


\section{Vibration Tests}

\subsection{Experimental method}

To evaluate the effects of the change in surface structure and the contact area difference of the solidified ionic liquids on the power generation performance, a vibration test was conducted in a glove box filled with argon gas. The evaluation system of the vibration test is shown in Fig. 7. The glass substrate deposited with the $\mathrm{Au} / \mathrm{Cr}$ film was fixed on a shaker (WaveMaker 01, Asahiseisakusyo Corp.). Then, the solidified ionic liquid was placed on the shaker with the cation side up. The glass substrate with an ITO film used as the upper electrode was in contact with the solidified ionic liquid during the excitation of the shaker. The inclination of the upper electrode substrate was adjusted horizontally so that the solidified ionic liquid and ITO electrode were in contact parallel to each other. Then, a triangular wave voltage was input from a function generator (33500 B, Keysight Technologies) to the vibration shaker. When the shaker table was vibrated, the solidified ionic liquid repeatedly induced contact/release states with the upper electrode.

The voltages generated at the contact/release moments and the displacement of the lower electrode on the shaker table measured using a laser displacement meter (LK-G $3000 \mathrm{~V}$, Keyence) were recorded using an oscilloscope (DSOX 3054 T, Keysight Technologies). The load resistance for measuring the output voltage was $1 \mathrm{M} \Omega$.

Before the vibration test, the contact state was adjusted so that the upper electrode and solidified ionic liquid were lightly in contact with each other in the experimental setup. Then, an offset voltage was applied to the shaker to provide a constant gap between the upper electrode and the sample surface. Hence, the solidified ionic liquid periodically repeatedly induced the contact/release states with the upper electrode. Here, when a voltage higher than

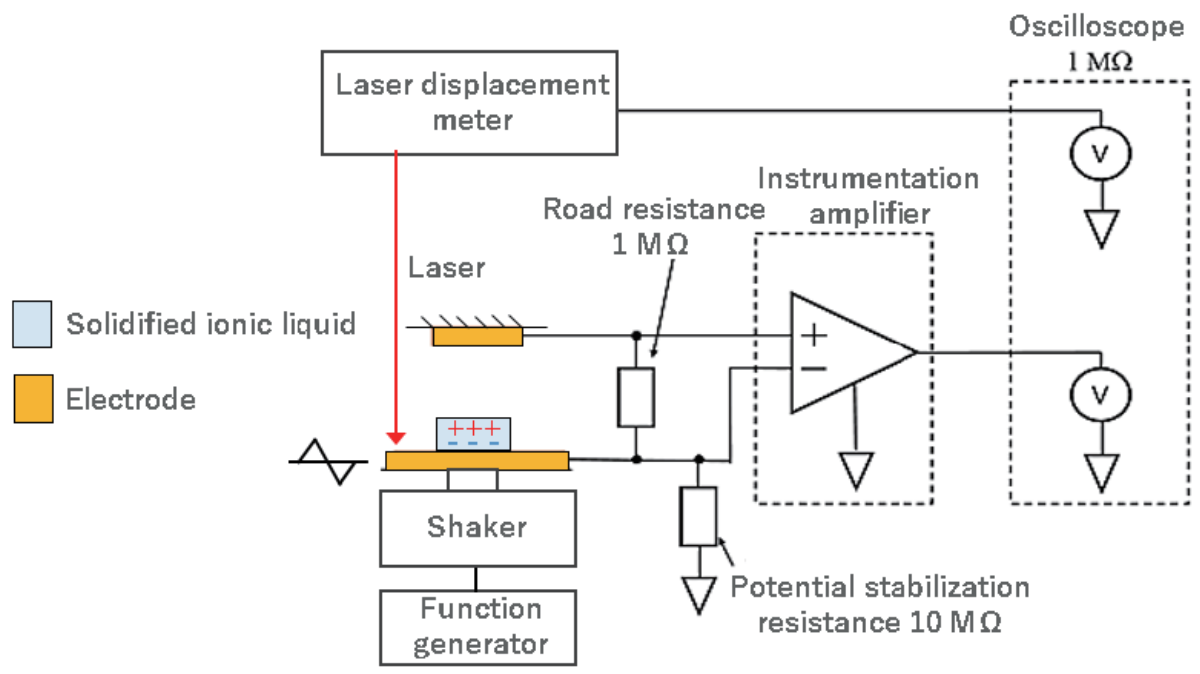

Fig. 7. (Color online) Experimental setup and evaluation circuit for vibration test. 
the offset value was input to the shaker, a slip occurred in the mechanism inside the shaker when the solidified ionic liquid and upper electrode were in contact. During the slip, the surface of the solidified ionic liquid was kept pushed by a force of $9.8 \mathrm{~N}$, which is the maximum excitation force of the shaker. We investigated the effect of frequency on the power generation performance at excitation frequencies of 10 and $50 \mathrm{~Hz}$ by the vibration test.

\subsection{Results and discussion}

Figures 8(a) and 8(b) show the displacement of the shaker table measured using the laser displacement meter, and Figs. 8(c)-8(f) show the output voltage waveforms of samples \#1 and \#4. In Figs. 8(a) and 8(b), the $0 \mathrm{~mm}$ displacement indicates the state where the electrode and solidified ionic liquid were in contact. Here, the displacement waveform in Fig. 8(b) was distorted by the impact of contact. Figures $8(\mathrm{c})-8(\mathrm{f})$ show the output voltages with time

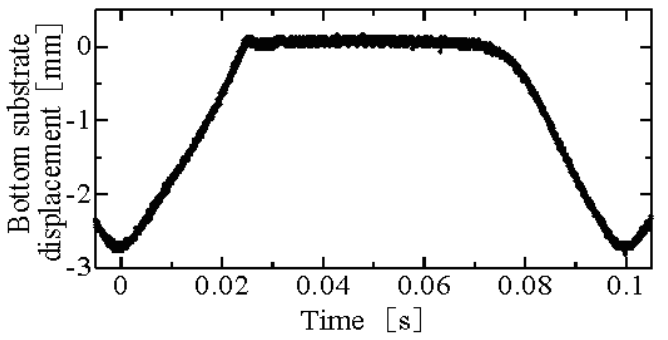

(a)

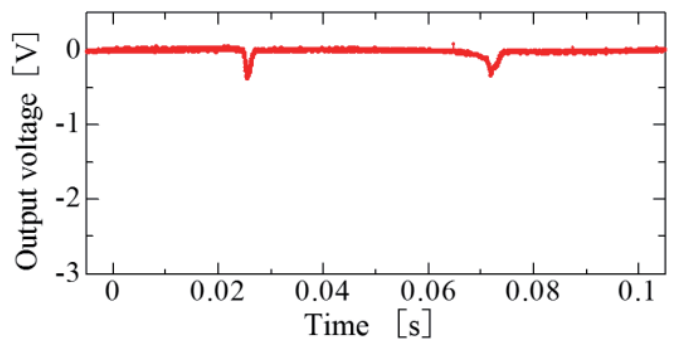

(c)

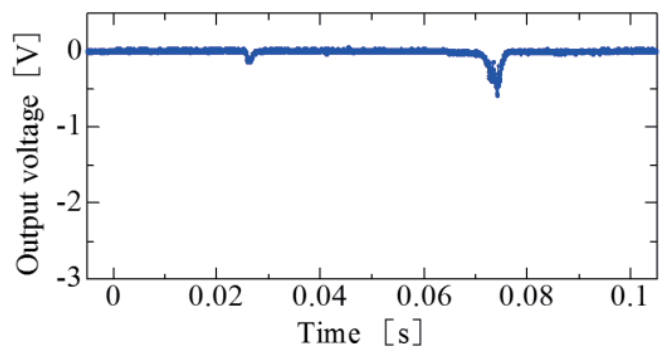

(e)

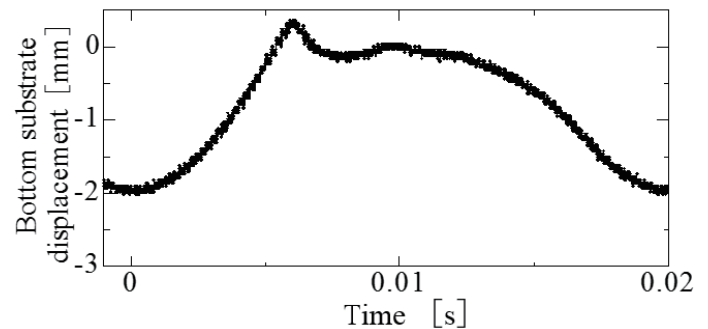

(b)

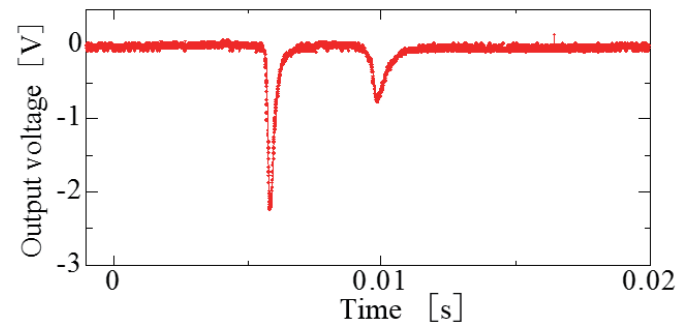

(d)

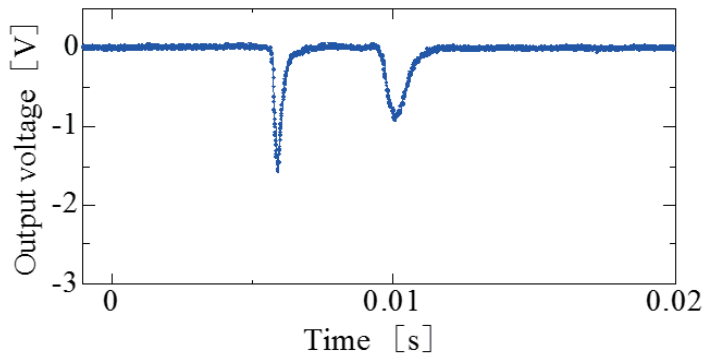

(f)

Fig. 8. (Color online) Time history response of shaker's bottom substrate displacement and output voltage. (a) Displacement of the shaker bottom substrate at $10 \mathrm{~Hz}$, (b) displacement of the shaker bottom substrate at $50 \mathrm{~Hz}$, (c) output voltage of sample \#4 at $10 \mathrm{~Hz}$, (d) output voltage of sample \#4 at $50 \mathrm{~Hz}$, (e) output voltage of sample \#1 at 10 $\mathrm{Hz}$, and (f) output voltage of sample \#1 at $50 \mathrm{~Hz}$. 
response. According to the voltage waveforms, the output voltages were generated at contact/ release moments between the electrode and the sample surface.

Figure 9 shows the maximum output voltage ratio of each sample with different surface structures at frequencies of 10 and $50 \mathrm{~Hz}$. The vertical axis shows the maximum output voltage of each sample normalized by that of sample \#1. The maximum output voltage of sample \#2 is 2.59 times larger than that of sample \#1. The maximum output voltage ratio is similar to the surface area ratio of the samples, 2.77. Thus, increasing the contact area between the solidified ionic liquid and the electrode is effective in improving the power generation performance.

In addition, sample \#3 with nanosized unevenness showed a 1.53 times larger output voltage than sample \#1 with a flat surface. Since the nanosized unevenness of the surface increased the contact area, the output voltage was improved. Samples \#4-\#6 with 3D microstructures obtained lower output voltages than sample \#1. By observing the deformation of the solidified ionic liquid from the top ITO glass substrate, the solidified ionic liquid was found to be rigid and not deformed sufficiently under the applied pressure in the experiment. It seems that only the upper surface of the 3D microstructure was in contact with the top electrode. In the case of sample $\# 5$, assuming that the contact occurs only on the upper surface of the cylindrical structure, the contact area is 0.17 times smaller than that of sample \#1. However, the measured voltage was 0.36 times smaller than that of sample \#1. Since the charge density of the cylindrical structure is increased by the electric charge fixed on the side wall of the microstructure, sample \#5 achieved a higher power generation performance with a unit area and the difference in output voltage ratio was generated. Moreover, sample \#4 showed a higher voltage than sample \#6. This is because sample \#4 is more flexible to deform and has a larger surface area to fix the electric charge than sample \#6. These results demonstrate that more

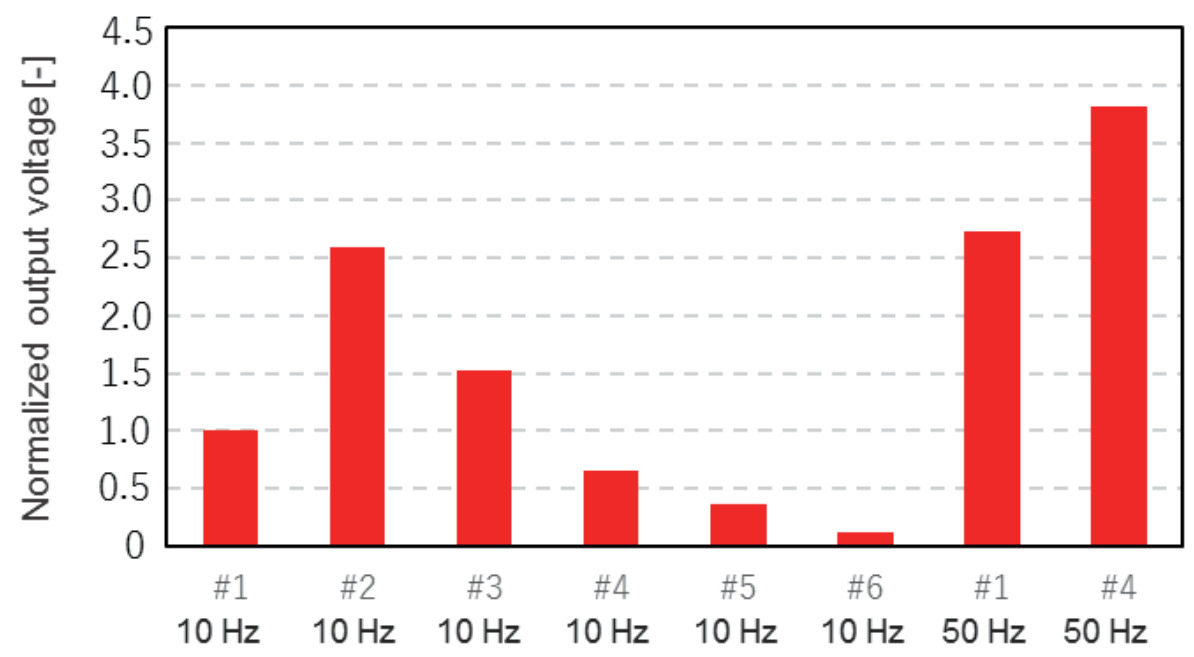

Fig. 9. (Color online) Normalized output voltage of each sample. Here, the output voltages are normalized by the value of sample \#1 with a flat surface. The frequency under the sample number shows the excitation frequency. 
deformable materials and structures with larger surface and side wall areas improve the power generation of the VEH with the solidified ionic liquid.

The change in power generation performance by the excitation frequency is discussed using the output voltage waveform in Fig. 8. The voltage in the contact state of sample \#4 was larger than that of sample \#1 at both frequencies of 10 and $50 \mathrm{~Hz}$. In particular, the maximum output voltage of sample \#4 at $50 \mathrm{~Hz}$ exceeded that of sample \#1. Since the concentrated load was generated in the microstructure, the piezoelectric effect increased with the maximum strain. On the other hand, the output voltage of sample \#4 in the release state was smaller than that of sample \#1. This indicates that the microstructure did not deform sufficiently, thus the contact area decreased. Therefore, the effects of electrostatic induction and triboelectric charge seem to be reduced. Also, in Fig. 9, the output voltage increases regardless of the surface structure with an increase in excitation frequency. The changes in gap between the top electrode and the solidified ionic liquid, and the strain rate of the solidified ionic liquid are faster with the increase in excitation frequency. Thus, both the electrostatic induction and the piezoelectric effect seem to be increased.

To compare the total power per oscillation at the frequency of $50 \mathrm{~Hz}$, the total power $P$ generated from the contact/release state is derived as

$$
P=\int_{0}^{T} P(t) d t=\int_{0}^{T} \frac{V(t)^{2}}{R} d t
$$

where $V(t)$ is the voltage generated as a function of time, $T$ is the time from the contact/release state, and $R$ is the load resistance. The total amount of power was derived as $1.26 \mathrm{~nJ}$ for the VEH with a 3D microstructural surface (sample \#4) and $0.95 \mathrm{~nJ}$ for the VEH with a flat surface (sample \#1). The VEH with 3D microstructures achieves a 33\% improvement in power generation performance at the frequency of $50 \mathrm{~Hz}$.

In the vibration test, a force of $9.8 \mathrm{~N}$ was repeatedly pushed to the solidified ionic liquid, but the microstructure was not broken. The common VEHs using MEMS technology suffer from poor durability due to miniaturization, but the solidified ionic liquid is very robust and applicable to more random vibration as an energy source compared with conventional VEHs. Therefore, the solidified ionic liquid with a microstructure is suitable for the VEH.

\section{Conclusions}

In this paper, we propose a novel forming method to simultaneously perform the solidification, polarization, and microstructure transfer of a solidified ionic liquid using a mold with 3D micropatterns fabricated by 3D lithography. We applied the solidified ionic liquid with a 3D microstructure to improve the output power without increasing the footprint of a VEH. The power performance of the VEH using the solidified ionic liquid was improved by increasing the contact area between the solidified ionic liquid and the electrode. 
In addition, 3D microstructures were not deformed sufficiently in the contact state at a frequency of $10 \mathrm{~Hz}$, and the VEH with 3D microstructures obtained a small contact area. Thus, the output power of the VEH with 3D microstructures was lower than that of the VEH with a flat surface. On the other hand, the VEH with 3D microstructures at the excitation frequency of $50 \mathrm{~Hz}$ achieved a higher output power than that with a flat surface because of the piezoelectric effect increase. In conclusion, a higher power generation performance is obtained for the solidified ionic liquid using a more deformable 3D microstructure.

\section{Acknowledgments}

This work was partly supported by JSPS KAKENHI Grant Number JP17H03196, JST PRESTO Grant Numbers JPMJPR15R3 and JPMJPR17R3, and Center for Nano Lithography \& Analysis, The University of Tokyo, supported by MEXT, Japan.

\section{References}

1 D. Bandyopadhyay: J. Sen: Wireless Pers. Commun. 58 (2011) 46. https://doi.org/10.1007/s11277-011-0288-5

2 S. Kaminaga: Sens. Mater. 30 (2018) 723. https://doi.org/10.18494/SAM.2018.1814

3 S. Priya and D. J. Inman: Energy Harvesting Technologies (Springer, Boston, 2009) p. 3. https://doi. org/10.1007/978-0-387-76464-1

4 N. Shiri, H. Veadi, and H. N. Oscuii: Sens. Mater. 30 (2018) 1319. https://doi.org/10.18494/SAM.2018.1740

5 W. S. Chen, C. M. Cheng, B. Y. Liao, Y. L. Chang, and H. Y. Wang: Sens. Mater. 30 (2018) 587. https://doi. org/10.18494/SAM.2018.1827

6 L. Mo, X. Lu, Z. Feng, and W. Hua: Sens. Mater. 29 (2017) 1353. https://doi.org/10.18494/SAM.2017.1612

7 G. Li, B. Yang, C. Hou, G. Tang, J. Liu, X. Chen, X. Wang, and C. Yang: Sens. Mater. 29 (2017) 1723. http:// doi.org/10.18494/SAM.2017.1586

8 S. P. Beeby, M. J. Tudor, and N. M. White: Meas. Sci. Technol. 17 (2006) R175. https://doi.org/10.1016/ j.nanoen.2013.08.002

9 G. Zhu, P. Bai, J. Chen, and Z. L. Wang: Nano Energy 2 (2013) 688. https://doi.org/10.1016/j.nanoen.2013.08.002

10 A. S. Holmes, D. A. Howey, A. Bansal, and D. C. Yates: Proc. Power MEMS (2010) 115. https://spiral.imperial. ac.uk/bitstream/10044/1/6127/1/PowerMEMS2010.pdf

11 Y. Lauw, M. D. Home, T. Rodopulos, A. Nelson, and F. A. M. Leermakers: J. Phys. Chem. B 114 (2010) 11149. https://doi.org/10.1021/jp105317e

12 C. Sano, H. Mitsuya, S. Ono, K. Miwa, H.Toshiyoshi, and H. Fujita: Sci. Technol. Adv. Mater. 19 (2018) 317. https://doi.org/10.1080/14686996.2018.1448200

13 T. Suzuki, H. Kotera, I. Kanno, and D. Hiramaru: U.S. Patent 8,871,433 (2014).

14 T. Tsukamoto, Y. Umino, S. Shiomi, K. Yamada, and T. Suzuki: Sci. Technol. Adv. Mater. 19 (2018) 660. https://doi.org/10.1080/14686996.2018.1508985

15 T. Tsukamoto, Y. Umino, K. Hashikura, S. Shiomi, K. Yamada, and T. Suzuki: J. Vis. Exp. (in press). https:// www.jove.com/video/59067/a-polymer-based-piezoelectric-vibration-energy-harvester-with-3d

16 H. Ueno, K. Yamada, and T. Suzuki: Micromachines 9 (2018) 681. https://doi.org/10.3390/mi9120681

\section{About the Authors}

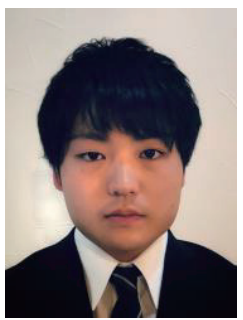

Taiki Iida received his B.S. degree from Gunma University, Japan, in 2018. Now he continues to pursue his M.S degree in the Division of Mechanical Science and Technology at the same university. His research interests are in MEMS and energy harvesting for IoT. (t181b007@gunma-u.ac.jp) 


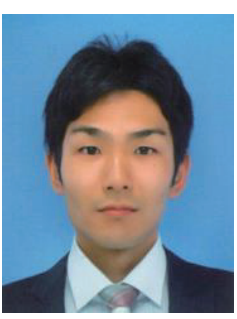

Takuya Tsukamoto received his B.S. degree from Gunma University, Japan, in 2017. Now he continues to pursue his M.S degree in the Division of Mechanical Science and Technology at the same university. His research interests are in MEMS and energy harvesting for IoT.

(t13305020@gunma-u.ac.jp)

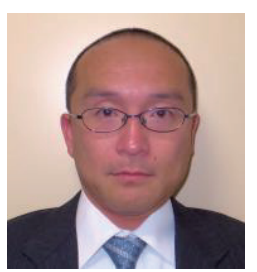

Kazumoto Miwa received his B.S. and M.S. degrees from Tokyo Institute of Technology, Japan, in 1996 and 1999, respectively. His research interests are in chemical engineering. (miwa@dentec.co.jp)

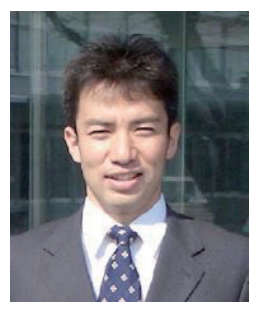

Shimpei Ono received his B.S., M.S, and Ph.D. degrees from Tokyo Institute of Technology, Japan, in 1996, 1998, and 2006, respectively. From 1998, he has been a research scientist at Central Research Institute of Electric Power Industry. From 2012, he has been a visiting professor at Grenoble-Alps University, France. His research interests are in solid-states physics, electrochemistry, and sensors. (shimpei@criepi.denken.or.jp)

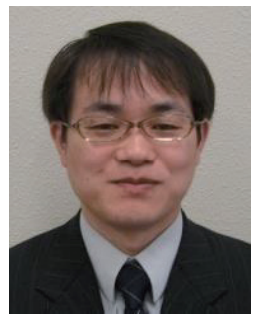

Takaaki Suzuki received his B.E. and M.E. degrees from Gunma University, Japan in 1998 and 2000, respectively. He also received his Ph.D. degree from Kyoto University, Japan in 2003. From 2004 to 2008, he was an assistant professor at Kyoto University. From 2008 to 2015, he was an associate professor at Kagawa University. From 2015 to 2018, he was an associate professor at Gunma University. Since 2018, he has been a professor at the Division of Mechanical Science \& Technology, Gunma University. Since 2015, he has also been a PRESTO researcher at Japan Science and Technology Agency. His research interests include the development of novel microfabrication technology, microfluidic systems for gene and cell analyses, and an energy harvester for IoT. He is a member of IEEE, the Japan Society of Mechanical Engineers, the Institute of Electrical Engineers of Japan, and so on. (suzuki.taka@gunma-u.ac.jp) 